\title{
El Banco Mundial y la política educativa para Colombia y Brasil
}

\author{
The World Bank and the Educational Policies \\ for Colombia and Brazil
}

\section{RESUMEN}

El artículo discute la ideología subyacente a las políticas evaluativas de Colombia y del Estado de Minas Gerais en Brasil. Estudio crítico apoyado en la pesquisa bibliográfica y el análisis documental, bajo la perspectiva del materialismo históricodialéctico y de los estudios históricos comparados. Los resultados de investigación demostraron que el Banco Mundial condiciona y determina las políticas educacionales de ambos sistemas escolares utilizando el discurso democrático de la gestión escolar. El artículo concluye que la lucha de este organismo financiero contra la pobreza consiste en utilizar la ideología gestiocrática a fin de ocultar y perpetuar las diferencias individuales y colectivas generadas por la miseria y la opresión, consecuencias del actual modelo de producción y consumo.

Palabras clave: política educativa, ideología, capitalismo

\section{Juan Francisco Remolina Caviedes*}

\section{ABSTRACT}

The article discusses the ideology underlying the evaluation policies of Colombia and the State of Minas Gerais in Brazil. A critical study supported by bibliographic research and documentary analysis, from the perspective of Historical-Dialectical Materialism and Comparative Historical Studies. The research results showed that the World Bank conditions and determines the educational policies of both school systems, using the democratic discourse of school management. The article concludes that the struggle of this financial institution against poverty consists of using the gestiocratic ideology in order to hide and perpetuate the individual and collective differences generated by misery and oppression, consequences of the current model of production and consumption.

Key words: educational policy, ideology, capitalism

\section{INTRODUCCIÓN}

Desde comienzos de la década de los noventa, el Banco Mundial (BM) viene politizando su intervención para con los estados deudores, sobre todo mediante la amplia participación en reformas antes consideradas de índole política (Burgos, 2007). Con ello, el BM rompe con el pretendido carácter técnico conferido desde su creación. La justificación: los impactos económicos derivados

\footnotetext{
* Facultad de Ciencias Humanas. Escuela de Idiomas. Grupo de Investigación Cultura y Narración en Colombia (CUYNACO); jremolinac@gmail.com
} 
de las decisiones políticas. Ahora bien, dado que el BM es quien evalúa tales impactos, esto le ha "facultado para intervenir en un casi ilimitado número de asuntos políticos" (Burgos, 2007, p. 23).

De esta manera, se infiere una interpretación del sentido que adquieren las decisiones y acciones del BM en materia de cooperación internacional. Según Pérez (2016), la expansión y consolidación del capitalismo en los denominados países subdesarrollados es el imperativo de la ayuda oficial para el desarrollo, de modo que la lucha contra la pobreza y la inequidad son acciones funcionales al servicio de esta lógica. Es así como, a través de los préstamos y recursos financieros concesionales (documentos estratégicos y misiones de asistencia técnica), el BM influye en el diseño y ejecución de la política pública de los estados prestatarios, respondiendo "a las demandas y necesidades propias de la acumulación de capital a escala planetaria” (Pérez, 2016, p. 120).

Por tanto, resulta evidente el sentido político de las misiones de asistencia técnica y financiera que el BM realiza en los estados prestatarios, la mayoría considerados en vía de desarrollo. Bajo esta perspectiva es necesario analizar la influencia del BM en el diseño y ejecución de las políticas educativas nacionales, dada la posibilidad de que conceptos como el de calidad educativa sean también funcionales a la lógica de expansión y consolidación capitalista, sobre todo, cuando ésta se legitima a través de documentos estratégicos y acuerdos internacionales que se acompañan con la ejecución de préstamos financieros.

La política educativa brasileña es ejemplo de esta influencia. En 2012, el BM elaboró el documento Achieving World Class Education in Brazil: The Next Agenda (Bruns et al., 2012), mediante el cual realiza un balance de los últimos veinte años de las políticas educacionales implementadas en Brasil. Según da Mota Junior y Maués (2014), en este texto se trazaron nuevas orientaciones encaminadas a dar continuidad a la regulación educacional fundada sobre la tríada del financiamiento per cápita, evaluación sistémica y gestión local, teniendo como referentes la prueba internacional Pisa y los estándares educativos de países miembros de la OCDE.

La crisis del sistema capitalista ha sido la puerta de entrada para el intervencionismo del BM y las principales reformas educacionales en Brasil, dado que la educación es concebida como 
parte del conjunto de reformas económicas (Figueiredo, 2009). El Projeto Nordeste para Educação Básica es un claro ejemplo, cuya cooperación internacional, bajo la modalidad de préstamos financieros, contaba con mecanismos contractuales compatibles con la expansión de los intereses del capital (da Cruz, 2003). A través de éste, el BM exigía la reducción del aparato estatal, la apertura de licitaciones internacionales y promovía la competitividad administrativa entre Estados. Es así como en la década de los noventa, los proyectos financiados por el BM contemplaron procesos de evaluación y competencia fundados sobre criterios de calidad, de racionalidad económica y productividad (Figueiredo, 2009).

Por otra parte, en Colombia, el BM financió un proyecto encaminado a aliviar el impacto que la crisis económica tenía sobre los pobres del país en 2001. Se hacía énfasis en proteger y promover la formación del capital humano de la población infantil más pobre (0 a 17 años) mediante el apoyo económico (subsidios) a estas familias, para mantener y mejorar sus inversiones en salud, nutrición y educación, dada la precariedad de los ingresos (Banco Mundial, 2001). Así, con el desembolso de 150 millones de dólares se pretendía frenar la crisis económica que amenazaba con reducir los gastos familiares en salud y educación, financiación que aspiraba aminorar a medida que el gobierno colombiano convirtiera su red de seguridad social en un programa moderno, bien dirigido y eficiente.

Asimismo, en 2007, el BM aprobó un crédito de 20 millones de dólares para financiar un proyecto de educación secundaria superior en el departamento de Antioquía. El objetivo consistió en mejorar, a largo plazo, la empleabilidad de los jóvenes y aumentar la competitividad de la fuerza laboral (Banco Mundial, 2015). Se financiaron planes de mejoramiento escolar que, entre otros aspectos, respaldaban el uso de resultados en las pruebas externas para proporcionar incentivos; la inversión en equipos, laboratorios, Tecnologías de Información y Comunicación (TIC) y bibliotecas; la capacitación de 2593 docentes en el currículo basado en competencias (una de las reformas centrales del proyecto) y la gestión escolar basada en la responsabilidad. 
Si bien Colombia ha recibido préstamos del BM desde 1949 (Londońo y Perry, 1985), llama la atención que sea casi nula la cantidad de trabajos de reflexión sobre la influencia del Banco en la política educativa colombiana. No fue posible encontrar estudios críticos en torno a los impactos de los subsidios ofrecidos en 2001, como tampoco respecto al proyecto financiado en el departamento de Antioquia. Podría pensarse que el influjo del BM en la configuración de la política educacional es inocua o más humanitaria que financista. Sin embargo, un análisis crítico al nuevo sistema de evaluación de la educación básica y media en Colombia tal vez aporte indicios en otro sentido, muy a pesar de no tener el apoyo directo del ente financiero.

El nuevo sistema colombiano de evaluación de la enseñanza básica y media está inspirado en el sistema evaluativo del Estado de Minas Gerais (Malaver, 2015). Modelo que a su vez nació de un proyecto educativo de mayor amplitud denominado ProQualidade (Araújo y da Silva, 2011), el cual en la década de los noventa recibió del BM un crédito de 150 millones de dólares (Grupo del Banco Mundial, 1994). De esta manera, el Sistema Mineiro de Avaliação da Educação Pública (Simave) consiguió incorporar orientaciones de organismos internacionales y políticas administrativas de carácter económico con el interés de garantizar la calidad educativa del Estado (Araújo y da Silva, 2011).

De modo que para el gobierno colombiano, Brasil se convirtió en referente obligado, dada la obtención inmediata de resultados favorables, según la ministra de educación de entonces. El Ministerio no estaba dispuesto a esperar diez ańos para mejorar los índices educacionales del país y el Simave era un modelo que ofrecía excelentes rendimientos casi al instante (Malaver, 2015).

Por todo lo anterior, además de la influencia del BM sobre la política evaluativa del Estado de Minas Gerais (Araújo y da Silva, 2011; Augusto, 2012; Santiago et al., 2013) y teniendo en cuenta que ésta sirve de inspiración al nuevo programa de evaluación en Colombia, resulta pertinente realizar un análisis crítico en torno a las posibles manifestaciones ideológicas que estarían permeando las políticas evaluativas de ambas realidades educacionales. Para tal efecto, asumo ideología como una estrategia consciente mediante la cual una clase hegemónica intenta disimular y ocultar 
las contradicciones de la realidad, presentándolas de manera incompleta (Lefebvre, 1975; Marx y Engels, 2014).

En tal sentido, propongo la siguiente pregunta de investigación desde la perspectiva teórico-metodológica del Materialismo Histórico Dialéctico (MHD) y utilizando el enfoque de los Estudios Históricos Comparados (EHC) (Ciavatta, 2009; M. C. Franco, 2000): en función de la lógica de expansión y consolidación capitalista que el BM promueve mediante su asistencia técnica y financiera, ¿qué tipo de ideologías subyacen a las políticas de evaluación de los sistemas educacionales de Minas Gerais y Colombia?

De esta forma, se implementaron las siete fases del MHD que son (Dussel, 2012): 1) lo existente, 2) la totalidad caótica, 3) las determinaciones abstractas, 4) la totalidad concreta construida, 5) las categorías explicativas, 6) la totalidad concreta histórica explicada y, por último, 7) la realidad conocida. Es decir, esta perspectiva teórico-metodológica propone un ascenso dialéctico de lo abstracto a lo concreto, del análisis a la síntesis para llegar a un retorno histórico explicativo mediante categorías conceptuales mutuamente determinadas.

Asimismo, los EHC se adoptan dada la exigencia de estudiar dos realidades distintas (internacionales). Este enfoque permite establecer diferencias entre contextos disímiles a partir de procesos históricos más amplios (comprender la historia como proceso) y posibilita la reconstrucción de tales divergencias como parte de una realidad compleja susceptible de transformación (historia como método) (Franco, 2000). De ese modo, se considera que en las Ciencias Humanas siempre estarán presentes los sujetos sociales que las producen y las ideologías que permean las concepciones sobre la verdad científica (Ciavatta, 2009).

De manera que se eligió una dimensión temporal con dos momentos, uno por cada país. El primero corresponde a los años 2010 y 2015, periodo que incluye la implementación del nuevo sistema evaluativo en la realidad colombiana. El segundo momento tiene que ver con el intervalo de tiempo entre los ańos 2000 y 2007, lapso que da origen al sistema de evaluación en el estado de Minas Gerais.

Con base en este interés y a la luz de los presupuestos episte-metodológicos asumidos, realicé un análisis documental ob- 
teniendo cuatro categorías histórico explicativas: gestión escolar y pedagógica; calidad educacional; cultura evaluativa y calidad profesoral. Ahora bien, dada la extensión de esta última categoría, decidí desarrollarla de manera exclusiva en otro trabajo. Por tanto, el presente artículo se limitará a la discusión de las tres primeras. A continuación, discutiré tres ideas claves del BM respecto al problema planteado: educación, evaluación y práctica docente; temas a partir de los cuales pareciera gravitar la política educacional y evaluativa de los contextos escolares estudiados. Luego, cada una de las categorías explicativas será debatida antes de abordar las consideraciones finales del trabajo, donde se intentará dar respuesta a la pregunta de investigación.

\section{EDUCACIÓN, EVALUACIÓN Y PRÁCTICA DOCENTE A LA LUZ DEL BANCO MUNDIAL}

A los ojos del BM (2017), la educación es motor de desarrollo, además de ser uno de los instrumentos más eficaces en la lucha contra la pobreza, para el mejoramiento de la salud, la igualdad de género, la paz y la estabilidad.

Si bien se han registrado importantes avances educativas durante la última década, el BM advierte que existen 250 millones de infantes en todo el mundo que no saben leer ni escribir, a pesar de haber asistido a la escuela. "La escolarización sin aprendizaje no es sólo una oportunidad desaprovechada, sino también una injusticia para los nińos que más la necesitan”, en definitiva, "es una falla moral” (Banco Mundial, 2017).

Orientar las políticas públicas de los países a fin de establecer reformas educativas que garanticen mejoras en el aprendizaje, es uno de los objetivos del BM. Entre las principales estrategias están: ofrecer financiamiento basado en resultados; respaldar evaluaciones de impacto rigurosas que establezcan indicadores comparables a nivel internacional sobre acceso educativo, tasas de rendimiento, resultados de aprendizaje y gastos del sector, entre otros; ajustar a nivel internacional las competencias de la clase trabajadora mediante la educación; establecer políticas docentes orientadas a la preparación, el apoyo y la gestión del profesorado de alto desempeño. 
Dos temas atraviesan el interés del BM en torno a las políticas educacionales: gestión escolar (Banco Mundial, 2010; Banco Mundial, 2014a; Banco Mundial, 2014b; Gropello, 2005) y calidad educativa (Kong et al., 2011). Mientras este último tiene que ver con el mejoramiento de los resultados de aprendizaje (Arcia et al., 2011), el primero se define como los grados de descentralización de la autoridad a nivel escuela, de manera que la responsabilidad y la toma de decisiones sobre los asuntos operativos institucionales son transferidos a las directivas, el profesorado, las familias y la comunidad en general (Barrera-Osorio et al., 2009).

Es así como escuela y profesorado deben rendir cuentas a las familias y a la sociedad por los resultados obtenidos en el marco de los indicadores nacionales, en las pruebas estandarizadas y en las evaluaciones del aprendizaje (Banco Mundial, 1996). Por tal razón, el BM recomienda implementar evaluaciones rigurosas a fin de establecer y documentar relaciones causales entre reformas educativas y resultados de aprendizaje.

Por otra parte, teniendo en cuenta que la responsabilidad es un atributo individual relacionado con el principio de libertad, cuyo fin es alcanzar mayor eficiencia y eficacia, para la sociedad es de sumo interés que los recursos y servicios prestados a una élite bien valorada sean debidamente retribuidos (Hayek, 2006).

De modo que si consideramos al profesorado siendo parte de esta élite, se entendería responsabilidad como instrumento de gestión orientado a mejorar la calidad educacional mediante lógicas de eficiencia y eficacia aplicadas al trabajo docente.

No en vano, el BM considera que la labor magisterial está relacionada de manera directa con la calidad de la educación. En consecuencia, formar docentes de alta calidad requiere selección, desarrollo y motivación personal-profesional (Bruns et al., 2012). Es por esto que, en consideración de los autores, debería existir una estructura salarial vinculada a la evaluación del desempeño docente para generar los incentivos necesarios y hacer de la docencia una carrera atractiva a profesionales de otras áreas.

En síntesis, el BM relaciona calidad educativa con resultados de aprendizaje medibles a través de exámenes e indicadores cuantitativos sensibles a la gestión escolar y a su dispositivo de responsabilidad, el cual se articula al trabajo docente mediante 
la supervisión del desempeño y la promoción de incentivos personales y profesionales.

\section{Gestión escolar y pedagógica}

Respecto a Minas Gerais, es importante indicar que su modelo de evaluación Simave comenzó como un sistema de evaluaciones censales durante el gobierno de Itamar Franco (1999-2003), para luego ser incorporado al programa Choque de Gestão del primer mandato de Aécio Neves (2003-2006), lo que cambiaría sensiblemente la naturaleza de las pruebas y los tipos de consecuencias para los sujetos docentes (Franco, 2016).

Este programa de gobierno, bajo el financiamiento y directrices del BM, implementó prescripciones económicas traducidas en un asunto de austeridad fiscal orientado a la construcción de un desarrollo económico equilibrado, mediante la reducción de gastos estatales (de Brito, 2013).

El Estado se reformó y afianzó su interés por supervisar las acciones que garantizaban la eficiencia y eficacia del sistema, bajo la lógica de la maximización de los resultados y la minimización de los gastos (Alves, 2006). De modo que las prácticas implementadas procuraron reformular el modelo de gestión de los recursos humanos, más allá de fortalecer alianzas con el sector privado en la prestación del servicio público, donde la evaluación y análisis del desempeńo se tornaron en elementos claves dentro de esta nueva lógica administrativa, explica la autora.

De esa forma, la evaluación del desempeño individual docente, basada en mecanismos de premiación y castigo, apareció como dispositivo estratégico orientado a la gestión del recurso humano en el Estado de Minas (Alves, 2006). Así, vinculado al modelo administrativo Choque de Gestão, el Simave implementó dispositivos de compensación a la productividad como estrategia para garantizar la calidad educacional (Franco, 2016).

En relación con la política educacional colombiana, para $\mathrm{Mi}$ ñana (2010), las intenciones de las últimas reformas han sido claras: la educación pública debe ser entendida como una educación mínima (no básica) para los más pobres, administrada por el sector privado y las actividades escolares sumidas en la lógica 
del mercado. Es así que durante el gobierno de Andrés Pastrana (1998-2002) las evaluaciones estandarizadas se volvieron obligatorias para todas las escuelas del país, agrega el autor.

Ferro et al. (2007) interpretaron estas reformas como consecuencia de las políticas mundiales de la economía de mercado dentro del fenómeno de la globalización, que transformó los dispositivos de gestión gracias al desarrollo del capitalismo (la revolución informática y microeconómica) (Abraham, 2002).

De esa manera, la gestión educacional como conjunto de metodologías, ideologías y prácticas orientadas a la administración y dirección, también pareció incorporar la ideología empresarial. El ámbito escolar fue permeado de la terminología administrativa, con lo cual la gestión pedagógica y administrativa fueron casi indivisibles. El control racional resultaría impuesto sobre la vida institucional y personal con los dispositivos de gestión, mudando en dirección del desarrollo de las fuerzas productivas. La eficiencia se transformaría en aplicabilidad de parámetros medibles, de los cuales también haría parte el producto del trabajo evaluado en las pruebas estandarizadas; de ahí, la sobrevivencia escolar comenzaría a producirse por medio de la gestión pedagógica y administrativa (Marin et al., 2005).

El interés parecería orientarse a organizar la vida personal e institucional a partir de la lógica neoliberal, en la cual el lucro, además de económico, sería político e ideológico. Con este fin, la escuela aplicaría la racionalidad empresarial mediante la gestión pedagógica, principal dispositivo de poder y control social del cual hace parte la evaluación, tal como afirman los autores.

Según de Carvalho (2009), esta nueva forma de gestión garantizaría la apropiación del fondo público a manos del capital privado, indicando que este nuevo modelo asumido por el Estado subordinó las instituciones públicas a la lógica del mercado (competencia, valorización del cliente consumidor, énfasis en los resultados, etcétera).

Así, las actuales políticas educacionales, orientadas a organizar el cotidiano escolar como estructuras que generan patrones conductistas, parecen sustituir todas las corrientes pedagógicas emancipadoras de antaño (Marin et al., 2005). He denominado ideología gestiocrática a este tipo de gestión, que propende a la organización social en torno a las leyes del mercado. 
La ideología gestiocrática es una racionalidad técnico-instrumental que, en el campo educativo, bajo la égida del neoliberalismo, promovería ideológicamente una reproducción alienada de la gestión escolar sobre los principios pragmáticos que fundamentan la lógica administrativa empresarial basada en criterios de eficacia y eficiencia. Esta manifestación ideológica actuaría de dos maneras: induciendo formas de intimidación (premios y castigos) y asociando autorrepresión con eficiencia y eficacia escolar. Una estrategia consciente -por tanto, ideológica- mediante la cual las contradicciones socioeconómicas serían excluidas del ámbito escolar al momento de someter la compleja realidad educativa a la mera cuantificación de sus resultados.

De esa manera, la realidad escolar no sería problematizada sino juzgada, desconsiderando el conflicto social mediante la búsqueda de interpretaciones periciales, de fácil transferencia y difícilmente vinculadas a las relaciones materiales del entorno, donde la evaluación se convertiría en otra sugerencia técnica de ajuste.

\section{Calidad educacional}

En la Constitución de Colombia, la primera y casi única referencia al concepto de calidad educativa aparece como responsabilidad del Estado determinada por acciones de inspección y vigilancia (Art. 67). Asimismo, el Estado espera que una educación de calidad ayude a formar mejores seres humanos con ciertos valores ciudadanos. En tal sentido, vincula calidad educativa con la Prueba Saber, ya que, a su parecer, "la evaluación mejora la calidad educativa" (Colombia, 2016).

Esto justifica la implementación de un indicador de calidad. El Índice Sintético de Calidad Educativa (ISCE) es una idea inspirada en el Índice de Desenvolvimento da Educação Básica (IDEB) de Brasil. El propósito es medir la calidad de la enseñanza colombiana en los niveles básico y medio. El ISCE está compuesto de cuatro variables: progreso, eficiencia, desempeño y ambiente escolar; que combinan resultados de exámenes estandarizados con datos contextuales.

Sin embargo, a diferencia del IDEB, el ISCE carece de sustento legal. No existe ley, decreto o resolución que lo implemente de 
manera directa y formal. Dentro de la normatividad existente, el Decreto No. 0325 de 25 de febrero de 2015 y la Resolución No. 15883 de septiembre 28 de 2015, se refieren de manera tangencial al ISCE. El primero, para establecer el Día de la Excelencia Educativa, mientras el segundo regula las tarifas de matrícula y pensión de las escuelas privadas en función de este indicador.

Tampoco fue posible determinar claramente la fundamentación conceptual del ISCE. No hay evidencias teóricas ni empíricas para sustentar una función de calidad basada en los atributos aludidos, más aún cuando no existe claridad sobre los procesos de medición y clasificación (Zambrano, 2015). Lo cual contrasta con las políticas que dieron origen al IDEB brasileño.

Según la Constitución de Brasil, la garantía de un patrón de calidad es uno de los principios fundamentales de la enseńanza (Art. 206), cuya asistencia técnica y financiera es responsabilidad de la Unión (Art. 211). De igual modo, es uno de los objetivos del sistema educacional en régimen de colaboración, regulado por la ley mediante el Plano Nacional de Educação (Art. 241).

En tal sentido, la Ley No. 9.394 de 1996 decreta garantizar patrones mínimos de calidad (Art. 4). Para esto, la Unión es responsable de implementar procesos de evaluación nacional en los diversos niveles de enseñanza (Art. 9). Mediante el Decreto No. 6.094/2007 el IDEB se establece como indicador objetivo de calidad. Alcanzar desempeños semejantes a los de países desarrollados es considerado uno de sus propósitos fundamentales. Esto ha intensificado el uso de evaluaciones estandarizadas en la gestión de las redes, además, el IDEB convirtió en transparente y objetivo el resultado del proceso educativo (Vidal y Vieira, 2011). La producción escolar consigue así ser representada por un dato cuantitativo de fácil comparación a nivel municipal, estatal y nacional.

Esto permite inferir que el concepto de calidad educativa tanto en Colombia como en Brasil no es ambiguo ni poco claro. $\mathrm{La}$ calidad es representada por un valor numérico, aunque existan diversas interpretaciones en torno a la efectividad de sus componentes y a la confiabilidad de su función.

Calidad educativa y aprendizaje, expresados como valores numéricos, permiten disponer de cuadros comparativos a la usanza internacional, lo que estaría promoviendo la fijación de padrones educacionales de desempeño a nivel global. Basta observar que la 
prueba Pisa condiciona indicadores y metas de calidad en ambos países.

Lo anterior providenció la implantación de políticas de responsabilidad o accountability. Uno de los objetivos de esta medición cuantitativa fue la producción de información para subsidiar sistemas de rendición de cuentas (da Cunha, 2013).

Aunque en Colombia las políticas de responsabilidad no aparecen claramente definidas ni materializadas, tal vez por cuenta de la inmadurez del sistema de evaluación escolar basado en indicadores de calidad, en el caso brasileño no es un asunto de menor cuantía. Desde la década de los noventa, el Estado de Minas Gerais aparece como uno de los entes federados provistos de un sistema de evaluación escolar financiado por organismos multilaterales. El BM ha aprobado y ayudado a adquirir préstamos destinados a beneficiar el sector educacional minero, entre otros, induciendo en el sistema escolar la incorporación de orientaciones y políticas administrativas de tipo económico, bajo el dilema de la calidad educativa. Así, se introdujeron mecanismos de responsabilidad mediante procesos de gestión que incluían premios a la productividad con significativa trascendencia en el trabajo docente. El profesorado comenzó a sentirse excluido, clasificado, responsabilizado y vigilado hasta el punto de oponerse al sistema evaluativo (Franco, 2016).

La tentativa de responsabilizar a cada escuela fue iniciada en Brasil a partir de la creación y puesta en práctica del IDEB (Freitas, 2015). Idea que, implementada en los EEUU, influyó la política pública brasileña, muy a pesar de los resultados negativos, aclara el autor.

Esta política pública de responsabilidad, fundada en evaluaciones de consecuencias fuertes, promovida por amplios sectores empresariales, tiene como misión la privatización de la educación pública (Emery, 2002). La autora enfatiza que este tipo de pruebas eliminaron el debate público sobre los fines de la educación, justificaron la desagregación del sistema escolar público y generaron un nuevo mercado educacional en beneficio del sector privado. Concluye que en este contexto las metas asociadas a la democracia fueron eliminadas, dejando a las escuelas la carga restricta de educar al alumnado para convertirse en operario altamente calificado para la Nueva Economía. 
Es por ello que a nombre de la sociedad civil, importantes gremios económicos, bajo el dilema de la participación y la calidad educativa, aparecieron como principales protagonistas en el diseño de la política educacional de ambos sistemas escolares. En el caso de Brasil, tal influencia se observó desde la configuración del Plano de Metas Compromisso Todos Pela Educação (Pmcte), que da vida al IDEB (da Paz y Raphael, 2010; Saviani, 2007), mientras en Colombia, agremiaciones como Empresarios por la Educación (ExE, 2012) y Todos por la Educación (Todos por la Educación, 2016) aún se esfuerzan por impulsar este tipo de iniciativas.

\section{Cultura evaluativa}

En ambos sistemas escolares se destaca una cultura evaluativa como estrategia de gobernanza, fundamentada en algo que denomino comparativismo globalizador, que tiene por base la expansión evaluativa.

Los postulados de las teorías de la modernización y del neomodernismo son el fundamento de esta cultura que utiliza ciertas formas de evaluación comparada para seducir a muchos países periféricos y semiperiféricos que luchan por el desarrollo económico (Afonso, 2013). Para el autor, esta forma de progreso está conectada a la agenda educativa de países capitalistas altamente desarrollados. Tales postulados son una falacia ideológica que esconde los verdaderos designios del capitalismo en el ámbito educacional, concluye.

De esta manera, las políticas educacionales comienzan a gravitar en torno de políticas de evaluación nacionales e internacionales de tipo comparativo, las cuales evidencian aspectos centrales como la conformación de pruebas estandarizadas, un sistema de responsabilidad (accountability), el pago por desempeño y la configuración de indicadores de calidad (Hypólito, 2013).

En palabras del autor, esto es una especie de reingeniería educacional basada en la lógica administrativa que busca aproximarse a los intereses de la sociedad del mercado como copias de modelos aplicados en otros países, tal como el programa No Child Left Behind (NCLB) del expresidente estadounidense George W. Bush. Modelo que afectó el contenido curricular, los exámenes 
de desempeño estudiantil, la formación docente y la distribución del presupuesto escolar.

Por esto, autoras como Santos (2013) hacen importantes cuestionamientos al mercantilismo globalizado de la educación y a sus prácticas evaluadoras. Considera imprescindible rebatir el tipo de valores que están en juego en el campo educacional, las formas de conducta y el ideal de persona a ser formada en y por la escuela, así como el contexto de mercantilización del conocimiento y de las relaciones sociales. Todo ello se constituye en pieza clave de una sociedad dominada por la cultura del consumo, que a su vez, substituye ideas de justicia y emancipación por la lógica de resultados medibles.

\section{CONSIDERACIONES FINALES}

En el marco de la asistencia técnica y financiera promovida por el BM, este artículo pretendió evidenciar el tipo de ideologías subyacentes a las políticas públicas de evaluación de los sistemas escolares de Minas Gerais y Colombia. La perspectiva teóricometodológica asumida (MHD) permitió ir de lo abstracto a lo concreto, del análisis a la síntesis, de lo existente caótico a la realidad explicada. Del mismo modo, el enfoque de los EHC posibilitó la lectura en paralelo de ambas realidades, superando el lenguaje formalizado y positivista del planeamiento económico y de los acuerdos internacionales, elementos consustanciales de la totalidad caótica analizada que desconsideran las relaciones de poder implícitas en la realidad educativa (Franco, 2000).

Un primer acercamiento deja ver que el BM en cuanto organismo financiero considera la educación como eje central del desarrollo social y económico de cada país. Bajo esta premisa concede créditos financieros. A cambio, sugiere la implementación de determinadas políticas públicas. Es el caso del Estado de Minas Gerais y la plataforma político-administrativa del denominado Choque de Gestão.

De esta forma, encontrar la autoridad representativa de las ideas globalizadas de la educación y la evaluación hizo posible la eliminación del carácter especulativo de estas concepciones al 
desmitificarlas y ubicarlas en vínculo directo con los procesos socioeconómicos de cada país.

Así, las políticas de evaluación evidenciaron en ambos países un fenómeno de doble transferencia. Primero de tipo cultural, con la influencia de una sociedad sobre otra; pero también de carácter político, mediante la participación de gremios económicos en la configuración de la política pública educacional.

De igual modo, tales directrices gubernamentales son parte de un fenómeno denominado comparativismo globalizador. El interés de estandarizar los fines y desempeños educacionales mediante estrategias de responsabilidad y gestión se ha convertido en una práctica internacional que apela a la evaluación como mecanismo de regulación y comparación. De manera que la práctica evaluadora se manifiesta como táctica útil para disimular contradicciones socioeconómicas.

De esta manera, la calidad educativa resulta inducida por la lógica empresarial de la cuantificación y la medición. Bajo esta perspectiva se da mayor participación a la sociedad civil en general y, particularmente, a importantes grupos empresariales. Así, tal como Marin et al. (2005) afirman, a la escuela se le ha transferido la misión de reinventar y extender los significados dominantes de la actual sociedad capitalista.

En consecuencia, al intentar responder la pregunta de investigación, es posible argumentar que detrás del discurso democrático de la gestión escolar, aparece la ideología gestiocrática. Esta manifestación fenoménica permite una fuerte reproducción alienada de la gestión bajo los auspicios de la racionalidad técnico-instrumental que fundamenta la lógica empresarial basada en criterios de eficacia y eficiencia, siendo la responsabilidad (accountability) su principal dispositivo de control.

Por tanto, podemos concluir que la ideología gestiocrática, articulada orgánicamente a las políticas de evaluación de Colombia y Brasil (estado de Minas), es indicio de una estrategia generalizada por medio de la cual se pretende encubrir, en el contexto escolar, las actuales contradicciones socioeconómicas del sistema de producción capitalista en ambos países. Su objetivo principal es aproximar la educación a los intereses del mercado y del capi- 
tal, utilizando mecanismos de calidad, participación y responsabilidad.

Así, la lucha contra la pobreza liderada a nivel global por el $\mathrm{BM}$, consiste en ocultar y perpetuar las profundas diferencias individuales y colectivas generadas por la miseria y la opresión, consecuencias del actual modelo capitalista de producción y consumo.

\section{REFERENCIAS BIBLIOGRÁFICAS}

Abraham, T. (2002). La empresa de vivir. Buenos Aires: Editorial Sudamericana.

Afonso, A.J. (2013). Mudanças no Estado-avaliador: comparativismo internacional e teoria da modernizaçáo revisitada. Revista Brasileira de Educação, 18(53), 267-284.

Alves, A.C.T. (2006). As reformas em Minas Gerais: Choque de Gestão, avaliação de desempenho e alterações no trabalho docente. En Vi seminário da redestrado-regulação educacional e trabalho docente (pp. 1-13). Rio de Janeiro-RJ.

Araújo, A.B., y da Silva, M.A. (2011). O lugar do Sistema Mineiro de Avaliação da Educação Pública (Simave) na busca pela qualidade da educação no Brasil. Roteiro, 36(2), 205-224.

Arcia, G., Macdonald, K., Patrinos, H.A., y Porta, E. (2011). System Assessment and Benchmarking for Education Results. Washington DC: Grupo del Banco Mundial.

Augusto, M.H. (2012). Regulação educativa e trabalho docente em Minas Gerais: a obrigação de resultados. Educação e Pesquisa, 38(03), 695-709.

Banco Mundial. (1996). Prioridades y estrategias para la educación. Washington, DC: Banco Internacional de Reconstrucción y Fomento/Banco Mundial.

Banco Mundial. (2010). School Based Management (APL II). Proyectos y Operaciones. Banco Mundial.

Banco Mundial. (2014a). "Economics of Education". Brief. Recuperado de http://www.worldbank.org/en/topic/education/brief/economics-of-education 
Banco Mundial. (2014b). Proyecto: Fortalecimiento de la Autonomía de Gestión Escolar. Plan Indígena 2014. Banco Mundial. Banco Mundial. (2001). Report No. PID9939. Project Name: Colombia-Human Capital Protection. Washington DC. Recuperado de: http://documentos. bancomundial.org/curated/es/ 629171468749727880/pdf/ multi0page.pdf

Banco Mundial. (2015). Report No. ICRR00003198. implementation completion and results report (ibrd-74920) on a loan in the amount of US\$20 million to the department of Antioquia with the guarantee of the republic of Colombia for an upper secondary education project. Washington, DC. Recuperado de http://documentos. bancomundial.org/curated/es/ 485271468018057619/pdf/ ICR31980P052600Official0Use0Only090.pdf

Banco Mundial. (2017). Educación. Temas. Banco Mundial.

Barrera-Osorio, F., Fasih, T., Patrinos, H. A., y Santibáñez, L. (2009). Toma de decisiones descentralizada en la escuela. Washington, DC: Banco Internacional de Reconstrucción y Fomento/Banco Mundial.

Bruns, B., Evans, D., y Luque, J. (2012). Achieving World-Class Education in Brazil. Whasington DC: The International Bank for Reconstruction and Development/Banco Mundial.

Burgos, J. G. (2007). (2007). El Banco Mundial y la politización de su mandato. Análisis Político, 20(59), 3-23.

Ciavatta, M. (2009). Estudos comparados: sua epistemologia e sua historicidade. Trabalho, Educação e Saúde, 7(Suppl. 1), 129-151. https://dx.doi.org/10.1590/S198177462009000400007

Colombia. (2016). Evaluación. Bogotá. Recuperado de https:// www.mineducacion.gov.co/1759/w3-article-179264.html

Da Cruz, R.E. (2003). Banco Mundial e política educacional: cooperação ou expansão dos interesses do capital internacional. Educar (22), 51-75. Recuperado de http://www. scielo.br/pdf/er/n22/n22a03.pdf

Da Cunha e Melo, M.P. (2013). Sistemas de avaliação e reforma educacional. En A. Bauer y B.A. Gatti (Eds.), Vinte e cin- 
co anos de avaliação de sistemas educacionais no brasil (Vol. 2, pp. 27-41). Florianópolis: Insular.

Da Mota Junior, W.P., y Maués, O.C. (2014). O Banco Mundial e as Políticas Educacionais Brasileiras. Educação e Realidade, 39(4), 1137-1152. Recuperado de http://www.scielo. br/pdf/edreal/v39n4/10.pdf

Da Paz, F.M., y Raphael, H.S. (2010). O IDEB e a qualidade da educação no ensino fundamental: fundamentos, problemas e primeiras análises comparativas. Revista OMNIA Humanas, 3(1), 8-30.

De Brito, V.L. F.A. (2013). Efeitos negativos do "choque de gestão" na remuneração de professores em Minas Gerais. Fineduca, 3(6), 1-19.

De Carvalho, E.J.G. (2009). Reestruturação produtiva, reforma administrativa do Estado e gestão da educação. Educ. Soc., 30(109), 1139-1166.

Dussel, E. (2012). A produção teórica de Marx. São Paulo: Expressão Popular.

Emery, K. (2002). The Business Roundtable and Systemic Reform (Tesis doctoral). University of California. Davis, CA. ExE. (2012) + +10 años sumando a la calidad [Manual de software informático]. Bogotá D.C.

Ferro, J., Maldonado, Prieto, C.M., Díaz, y Quijano, L.F., Wilches. (2007). Economía, Globalización y Educación. Educación y Ciencia (10), 13-18.

Figueiredo, I.M.Z. (2009). Os projetos financiados pelo Banco Mundial para o ensino fundamental no Brasil. Educ. Soc., 30(109), 1123-1138.

Franco, K. (2016). Sistema Mineiro de Avaliação da Educação Pública (SIMAVE). Mestrado em Educação. Campinas, SP Programa de Pós-Graduação em Educação do Centro de Ciências Humanas e Sociais Aplicadas (CCHSA) da Pontifícia Universidade Católica de Campinas.

Franco, M.C. (2000). Quando nós somos o outro. Educ. Soc., 21(72), 197-230.

Freitas, L.C. (2015). IDEB. Avaliação Educacional-Blog do Freitas. Recuperado de https://avaliacaoeducacional. 
com/2015/02/02/ideb-como-uma-ideia-falida-influenciou-o-brasil/

Gropello, E.D. (2005). Análisis comparativo de la gestión centrada en la escuela en América Central. En Breve (72). Recuperado de http://documentos.bancomundial.org/curated/ es/542931468023460685/ pdf/342050SPANISH0revdf.pdf Grupo del Banco Mundial. (1994). Brazil-State of Minas Gerais [MOP]. Washington, DC. Recuperado de http://documentos.bancomundial.org/curated/es/ 612311468231287584/pdf/multi0page.pdf

Hayek, F.A. (2006). Los fundamentos de la libertad (7a ed.). Madrid: Unión Editorial.

Hypólito, A.M. (2013). Necessária meta-avaliação das políticas de avaliação. En A. Bauer y B.A. Gatti (Eds.), Vinte e cinco anos de avaliação de sistemas educacionais no brasil (vol. 2, pp. 211-227). Florianópolis: Insular.

Kong, L.S., Boon, G.C., Fredriksen, B., y Peng, T.J. (2011). Hacia un futuro mejor. Santiago de Chile: Academia Chilena de Ciencias.

Lefebvre, H. (1975). Lógica formal Lógica dialética. Río de Janeiro: Civilização Brasileira.

Londońo, J.L., y Perry, G. (1985). El Banco Mundial, el Fondo Monetario y Colombia: Análisis crítico de sus relaciones. Coyuntura Económica, XV(3), 209-243.

Malaver, C. (2015). Llegó la hora de que colegios pasen al tablero cada ańo. El Tiempo. Recuperado de http://www. eltiempo.com/bogota/nuevo-modelo-de-educacion-encolombia/ 15399375

Marin, L.F., Rios, Ocampo, J.J., Franco, y Serna, E., Giraldo. (2005). Critica y Educación. Armenia: Fussion Creativa.

Marx, K., y Engels, F. (2014). La ideología alemana. Madrid España: Ediciones AKAL.

Mińana, C. (2010). Políticas neoliberales y neoinstitucionales en un marco constitucional adverso. Propuesta Educativa Número 34, 2(19), 37-52.

Pérez, I.E. (2016). Los senderos de los organismos internacionales en la cooperación para el desarrollo: un panorama 
general sobre la evolución de las estrategias del Banco Mundial. Análisis Político, 29(88), 105-125.

Santiago, J.V., de Souza, L.N., Veríssimo, M., da Silva, M.A., y Silva, W.D.O. (2013). As políticas de avaliação sistêmica em um modelo de estado gerencial: implicaçôes para a avaliação da aprendizagem. Intersaberes, 8(16), 25-58.

Santos, L.L. (2013). A Avaliação em Debate. En A. Bauer y B. A. Gatti (Eds.), Vinte e cinco anos de avaliação de sistemas educacionais no brasil (vol. 2, p. 229-245). Florianópolis: Insular.

Saviani, D. (2007). O Plano de Desenvolvimento da Educação: análise do projeto do MEC. Educ. Soc., 28(100), 12311255.

Todos por la Educación. (2016). Recuperado de http://todosporlaeducacion.co

Vidal, E.M., y Vieira, S.L. (2011). Gestão educacional e resultados no Ideb: um estudo de caso em dez municípios cearenses. EAEE - Estudos em Avaliação Educacional, 22(50), 419-434.

Zambrano, M.F. (2015). Alcances e inconsistencias del Índice Sintético de Calidad Educativa (ISCE), diseńado y aplicado por el Ministerio de Educación de Colombia en el año 2015. Working Paper, 1-17. 